\title{
Muestreo en transecto de formaciones vegetales de fanerófitos y caméfitos (MIFC) (II): estudio de los sabinares de la Reserva Biológica de Doñana (RBD) (España)
}

\section{Transect sampling of vegetation formations of phanerophytes and chamaephytes (MIFC) (II): Study of the savin juniper woods of the Doñana Biological Reserve (DBR) (Spain)}

\section{Rafael Cámara Artigas*, Fernando Díaz del Olmo** y César Borja Barrera***}

\section{DISTRIBUCIÓN GENERAL DEL GÉNERO JUNIPERUS ${ }^{1}$}

El género Juniperus se localiza en la actualidad en la zona templada y subtropical en Europa, Próximo Oriente (Arabia y Yemen), Norteamérica hasta Centroamérica (Schnell, 1987; Mao 2010) y la Macaronesia (Ferreras, 1987). En Norteamérica hay 13 especies, en dos grandes marcos ecológicos de distribución: ambientes xéricos al suroeste y oeste, y ambientes fríos al norte del continente y en contacto con los bosques de gimnospermas (Mao, 2010).

Las formaciones monoespecíficas de bosques de sabinos constituyen comunidades originales del suroeste de Europa (Costa, 1998), norte de Africa y la Macaronesia. Su distribución general es circunmediterránea, a excepción de Juniperus communis L. subsp. communis que alcanza Asia central y septentrional y

* Departamento de Geografía Física y Análisis Geográfico Regional, Universidad de Sevilla (rcamara@us.es).

** Departamento de Geografía Física y Análisis Geográfico Regional, Universidad de Sevilla (delolmo@us.es).

*** Departamento de Geografía Física y Análisis Geográfico Regional, Universidad de Sevilla (cesarborja@us.es).

${ }^{1}$ Este trabajo es una contribución al Proyecto de investigación OAPN036/2008 del Plan Nacional de I+D+i, Subprograma Medio Ambiente y Ecoinnovación, Subsector Parques Nacionales y al Proyecto Integral A1/035520/11 del PCI Brasil de la AECI. 
América del Norte, y Juniperus sabina L. que se distribuye además desde el Mediterráneo a Europa central y oeste de Asia hasta el Himalaya (Rougerie, 1990; Costa, 1998). Las especies presentes en la Península Ibérica son Juniperus communis L. subsp. communis (enebro común), Juniperus communis subsp. hemisphaerica (C. Presl) Nyman (enebro), Juniperus communis subsp. alpina (Suter) elak. (enebro rastrero), Juniperus oxycedrus L. (enebro de la miera), Juniperus oxycedrus subsp. macrocarpa (Sm.) Ball (enebro marino), Juniperus phoenicea L. subsp. phoenicea (sabina negral), Juniperus phoenicea subsp. turbinata (Guss.) Nyman (sabina marina o mora), Juniperus sabina L. (sabina rastrera) y Juniperus thurifera L. (sabina albar).

Las subespecies de Juniperus phoenicea presentan dos distribuciones: una continental (subsp. phoenicea) y otra litoral (subsp. turbinata) (Costa, 1998). Ambas tienen una amplia valencia térmica y logran sobrevivir a situaciones de estrés hídrico muy acentuado, mayor que la especie J. thurifera L., aunque son más sensibles las subespecies a las heladas que ésta.

Paleobiogeográficamente las gimnospermas mantienen su presencia en la flora tropical, desde el Mesozoico colonizando desde el sudeste asiático (bosques de Pinus y Keteleeria) (Kryshtofovich, 1929, 1935; Rothe, 1947; Blasco, 1996), hasta el Himalaya con bosques de Abies, Juniperus, Picea y Tsuga (Rougerie, 1990, p. 144). En América su presencia se asocia con los bosques de Pinus de distribución septentrional en Norteamérica con el límite sur en Honduras, y en Sudamérica desde la Patagonia hasta alcanzar límite norte en la actual frontera de México con EEUU con Podocarpus y Araucaria (Schnell, 1986, Tomo 1, pp. 76 y 77). La distribución de las gimnospermas no es tan destacada en África, quedando reducido el género Juniperus al África Oriental (Somalia, Etiopía) (Schnell, 1977, Tomo 1, pp. 63-66). El género Juniperus en concreto se distribuye desde el Eoceno-Oligoceno en el cinturón de vegetación del Madren-Tethyan aprovechando el North Atlantic Land Bridge (NALB) y el Bering Land Bridge (BLB) (Mao, 2010).

La subespecie Juniperus phoenicea subsp. turbinata (Guss.) Nyman tiene un marcado carácter xerófilo ocupando suelos y formaciones superficiales arenosas, con horizontes edáficos desagregados, secos con alta conductividad, por lo que es identificada como una especie edafoxerófila-psammófila en los litorales, con un estatus de rara a en peligro (Pérez Latorre, 2001) en el piso termomediterráneo árido-semiárido de Andalucía. En la proximidad de la línea de costa Juniperus phoenicea subsp. turbinata (Guss.) Nyman es sustituida por Juniperus oxycedrus subsp. macrocarpa (Sm.) Ball (Pérez Latorre, 2001).

Las formaciones de Juniperus phoenicea subsp. turbinata (Guss.) Nyman (sabina mora) se distribuye en el Mediterráneo occidental y meridional en 
unidades geomorfológicas dunares de dominios bioclimáticos áridos, ascendiendo en su distribución hasta $2.200 \mathrm{~m}$, extendiéndose a lo largo de transversal de Marruecos hasta el Sinaí, cerca del Mar Rojo. En la orilla septentrional del Mediterráneo se localiza en Francia, Italia hasta 1.200 m, y más al este en Croacia y región costera de Bosnia-Herzegovina. Existen enclaves en Arabia Saudí y en Yemen, ocupando situaciones de estepas cálidas. También aparecen en la región Macaronésica (Madeira y Canarias). En las Islas Canarias es aún abundante en el Hierro y La Gomera, y está presente con menor superficie en Tenerife y La Palma, siendo muy reducida su presencia en Gran Canaria.

DistribuCión DE JUNIPERUS PHOENICEA SUBSP. TURBINATA (GUSS.) NYMAN EN LA RBD

Regionalmente Juniperus phoenicea subsp. turbinata (Guss.) Nyman se distribuye en el litoral meridional portugués (Algarve) y en el español desde Huelva hasta Almería. Conforma la vegetación potencial de arenales y sistemas de dunas estabilizadas del litoral de Andalucía. Particularmente el estudio de los sabinares de la Reserva Biológica de Doñana, tiene como precedentes los trabajos de Allier (1974, 1977), Ramírez (1977), Rivas-Martínez (1980), Bejarano (1997a, 1997b, 1994, 2004), López (2009) y Bejarano y Cámara (2010).

Los sabinares de Juniperus phoenicea subsp. turbinata (Guss.) Nyman geobotánicamente colonizan los arenales y sistemas de dunas fitoestabilizadas del Alto Manto Eólico Seco (AMEs), que junto al Alto Manto Eólico Húmedo (AMEh) y el Manto Eólico de Dunas Semiestables (MEDS) constituyen el Manto Eólico Litoral de El Abalario-Doñana (Borja, 1992, 1997; Borja y Díaz del Olmo, 1994, 1996; Borja et al., 2008; Montes, 1998; Zazo et al., 1999; Bejarano, 2010).

Fitosociológicamente son considerados como comunidades permanentes sobre substratos especiales pertenecientes a la geoserie edafoxerófila litoral termomediterránea iberoatlántica de los ecosistemas dunares (Valle, 2003) identificada con la asociación Osyrio quadripartitae-Juniperetum turbinatae, del piso bioclimático termomediterráneo seco-subhúmedo, acompañadas de enebro (Juniperus oxycedrus subsp. macrocarpa en el litoral, configurando la asociación sobre dunas fijas Rhamno oleoidis-Juniperetum macrocarpae. En concreto, los sabinares forman parte de la asociación Osyrio quadripartitae-Juniperetum turbinatae, en contraposición a los enebrales Rhamno oleoidis-Juniperetum macrocarpae, cuya ubicación corresponde al sector más expuesto a la influencia de la brisa del mar. La serie incluye otras comunidades: Rubio longifoliae-Corematetum albi, Artemisio crithmifoliae-Armerietum pungentis, y Lina- 
rion pedunculatae (Valle, 2003). Los jaguarzales (Halimio halimifolii-Stauracanthetum genistoidis), localmente denominados monte blanco, constituyen la etapa de sustitución de esta serie (Asensi y Díez, 1987).

\section{OBJETIVOS, MATERIAL Y MÉTODO}

Se aborda en el presente artículo la aplicación del Método de Inventario de Fanerófitos y Caméfitos (MIFC) para el estudio geobotánico de los sabinares de la Reserva Biológica de Doñana (RBD). Se han realizado 12 parcelas de 50x2 m organizadas en 5 transectos de $50 \mathrm{~m}$ en los enclaves del entorno de las lagunas de Charco del Toro y Navazo del Toro. La caracterización del método y sus técnicas han sido desarrolladas en un artículo anterior (Cámara y Díaz del Olmo, 2013).

APlicación Del MifC Al ESTUdio DE LOS SABINARES DE JUNIPERUS PHOENICEA SUBSP. turbinata (Guss.) NyMAN en la RESERVA BIOlógica de DoÑana (RBD)

\section{Estudios previos de los sabinares de la RBD}

El área de estudio comprende un sector de bosque de naves situado sobre la zona de cotos o dunas fitoestabilizadas, entre las lagunas de Charco del Toro y El Brezo al suroeste del Parque Nacional de Doñana, y al este de la Nave de Pedro Pérez en las proximidades del núcleo turístico de Matalascañas; más al norte un segundo sector se sitúa en el contacto entre el AMES y el AMEH en la proximidad de la laguna de Navazo del Toro, en el paraje conocido como Sabinar del Ojillo (figura 1).

Bejarano (2004) realizó un ensayo de tipología de los sabinares costeros de Andalucía occidental en función de los criterios de composición florística, abundancia-dominancia por especie (método fitosociológico), estructura (según el método de Bertrand) y riqueza florística de las leñosas acompañantes de la sabina. Por su parte Cámara y Bejarano (2010) realizaron una comparación de las mismas parcelas de sabinar situadas al norte del Charco del Toro confrontando los resultados entre las estudiadas con el método fitosociológico y el método Gentry.

López (2009) en su tesis doctoral aborda el estudio de la vegetación del manto eólico de Doñana desde el método fitosociológico, identificando como uno de los problemas la ausencia de aleatoriedad en los muestreos. Para obtener una buena significación estadística, utiliza el método multivariante de K-means (KM), para el análisis de los datos de las parcelas. Para poder detec- 
FIGURA 1

LOCALIZACIÓN DE PARCELAS DE SABINAR CHTV EN CHARCO DEL TORO (DETALLE ABAJO A LA IZQUIERDA) Y NVTV EN EL SABINAR DEL OJILLO AL SUROESTE DE LA LAGUNA NAVAZO DEL TORO (DETALLE ABAJO A LA DERECHA)
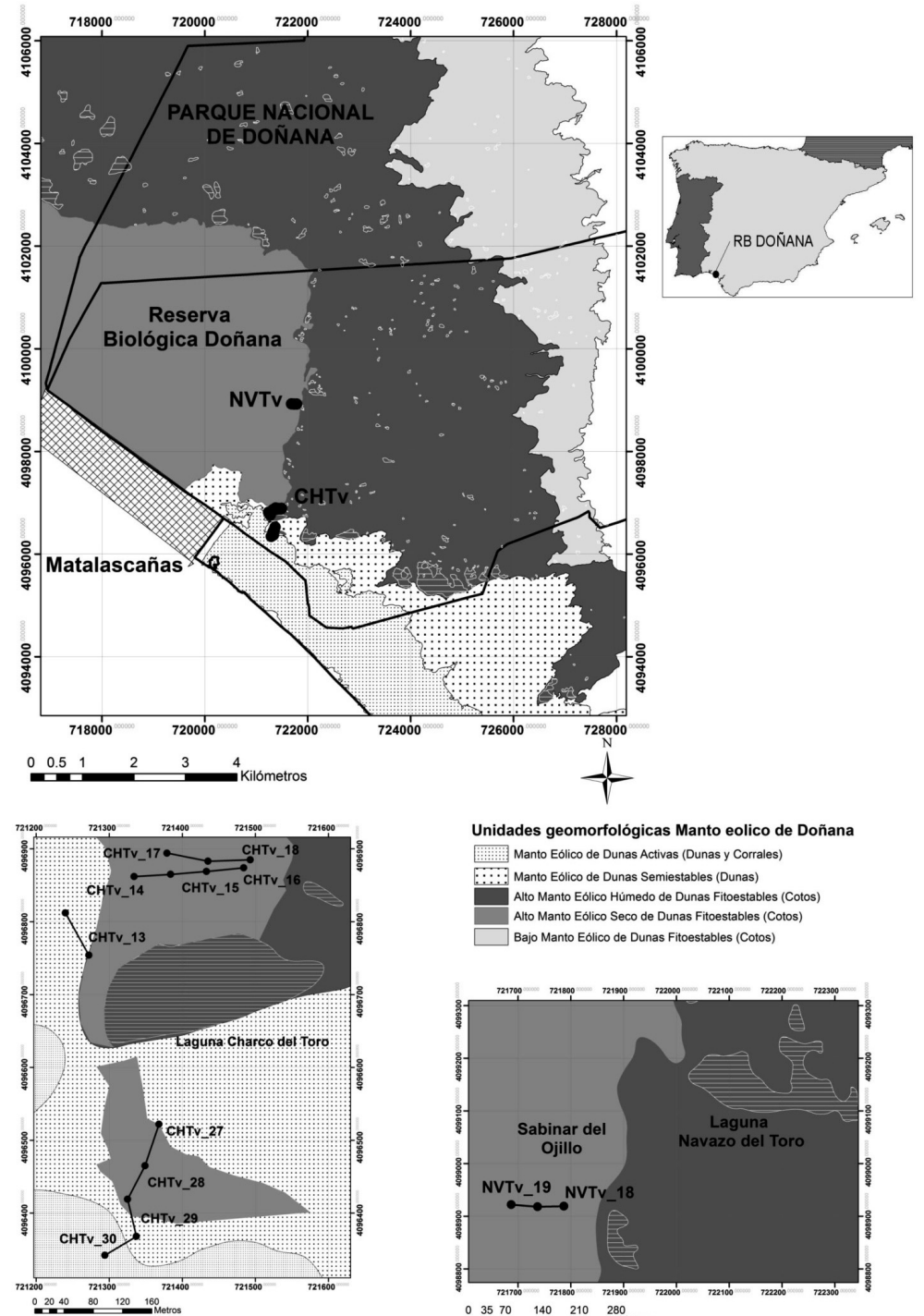

Unidades geomorfológicas Manto eolico de Doñana Manto Eólico de Dunas Activas (Dunas y Corrales) ::::: Manto Eólico de Dunas Semiestables (Dunas) Alto Manto Eólico Húmedo de Dunas Fitoestables (Cotos) Alto Manto Eólico Seco de Dunas Fitoestables (Cotos) Bajo Manto Eólico de Dunas Fitoestables (Cotos)

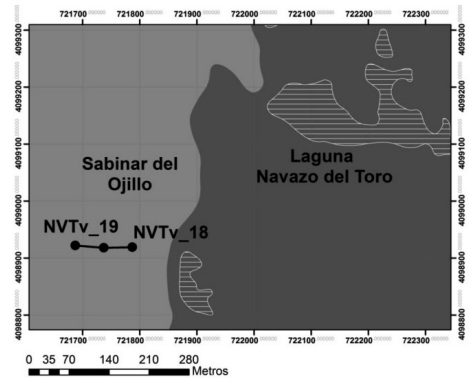

Fuente: elaboración propia. 
tar el mayor número posible de asociaciones realiza un análisis con el método partitivo Fuzzy C-Mean (FCM). Por último, con el objetivo de probar la validez o consistencia del modelo de clasificación aplica a los datos un análisis de similaridad con el método ANOSIM, identificando 23 grupos de los que 2, el 10 y el 16, incluye a formaciones vegetales con sabinos.

\section{Caracterización de las parcelas y sus elementos}

Hemos llevado a cabo 12 parcelas de $100 \mathrm{~m}^{2}$ (50 x $2 \mathrm{~m}$ ) cada una, en los sabinares próximos a las lagunas de Charco del Toro (10, con las siglas CHTv) y de Navazo del Toro (2, con las siglas NVTv) (figura 1 y tabla 1). En estas 12 parcelas se han muestreado 617 individuos pertenecientes a 17 familias, todas con 1 especie cada una, a excepción de Fabaceae (2), Lamiaceae (4) y Cistaceae (5), que son las familias que tienen mayor representación específica. En abundancia y cobertura la especie más importante es Juniperus phoenicea subsp. turbinata (Guss.) Nyman con 186 individuos, y con más de 30 indivi-

\section{TABLA 1}

GEOLOCALIZACIÓN DE LOS TRANSECTOS (DATUM WGS84 29N), FECHA DE

LEVANTAMIENTO Y ESTACIÓN (HÚMEDA: OTOÑO-INVIERNO; SECA: PRIMAVERA-VERANO) Y SI EL AÑO HA SIDO HÚMEDO (HUM) O SECO (SEC)

\begin{tabular}{lllllll}
\hline Transecto & X inicial & Y inicial & X final & Y final & fecha & Estación/año \\
\hline NVTv_18 & 721787 & 4098919 & 721737 & 4098918 & $14 / 04 / 11$ & Húmeda/HUM \\
NVTv_19 & 721737 & 4098918 & 721687 & 4098922 & $14 / 04 / 11$ & Húmeda/HUM \\
CHTv_13 & 721272 & 4096754 & 721240 & 4096812 & $20 / 11 / 09$ & Húmeda/HUM \\
CHTv_14 & 721334 & 4096862 & 721384 & 4096865 & $20 / 11 / 09$ & Húmeda/HUM \\
CHTv_15 & 721384 & 4096865 & 721433 & 4096869 & $20 / 11 / 09$ & Húmeda/HUM \\
CHTv_16 & 721433 & 4096869 & 721484 & 4096874 & $11 / 02 / 10$ & Húmeda/HUM \\
CHTv_17 & 721379 & 4096894 & 721435 & 4096883 & $05 / 03 / 10$ & Húmeda/HUM \\
CHTv_18 & 721435 & 4096883 & 721493 & 4096885 & $05 / 03 / 10$ & Húmeda/HUM \\
CHTv_27 & 721368 & 4096522 & 721349 & 4096465 & $19 / 02 / 10$ & Húmeda/HUM \\
CHTv_28 & 721349 & 4096465 & 721435 & 4096419 & $19 / 02 / 10$ & Húmeda/HUM \\
CHTv_29 & 721325 & 4096419 & 721337 & 4096368 & $19 / 02 / 10$ & Húmeda/HUM \\
CHTv_30 & 721337 & 4096368 & 721294 & 4096342 & $19 / 02 / 10$ & Húmeda/HUM \\
\hline
\end{tabular}

Fuente: elaboración propia. 
FIGURA 2

CURVA ESPECIES-ÁREA DE LA MUESTRA OBTENIDA DE 12 PARCELAS DE BOSQUE DE SABINO Y ASÍNTOTA DE LA CURVA SEGÚN EL MÉTODO DE CAIN (1938)

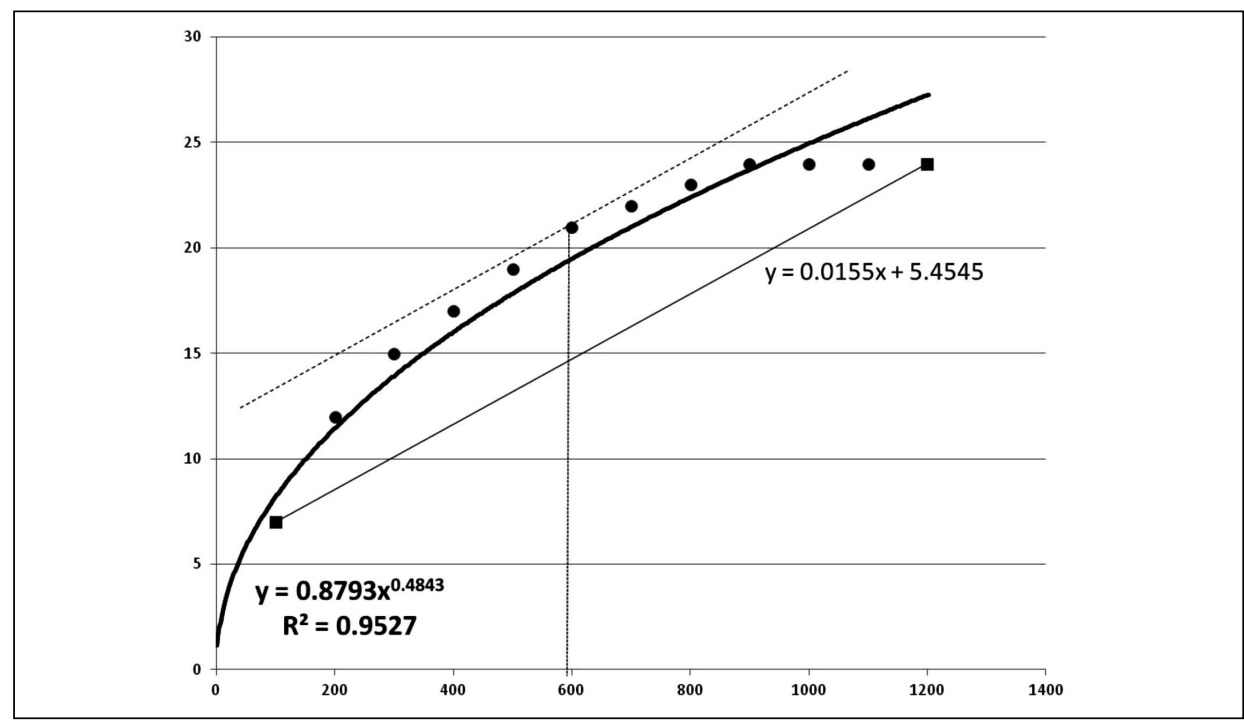

Fuente: elaboración propia.

duos se encuentran Cistus libanotis L., Cistus salviifolius L., Halimium halimifolium (L.) Willk. in Willk. \& Lange, Lavandula pedunculata (Mill.) Cav. y Rosmarinus officinalis L. Las familias con mayor abundancia son Cistaceae (190 individuos), Cupresaceae (186 individuos) y Lamiaceae (117 individuos).

La curva área-especies (figura 2) muestra que se alcanza la asíntota en torno a los $600 \mathrm{~m}^{2}$ de área inventariada, lo que conformaría el área mínima para el análisis de bosques de sabinar en la RBD, y nos confirma que con 12 parcelas de $100 \mathrm{~m}^{2}$ la unidad es suficientemente representativa para el estudio de los mismos. El valor elevado del exponente $z(0,48)$ muestra un crecimiento importante de la diversidad con el aumento del área muestreada. El área mínima se calcula por el procedimiento gráfico de Cain (1938), con la recta tangente a la curva paralela a la que une el valor máximo y mínimo de la serie.

La distribución de frecuencias absolutas (figura 3) de las clase de presencia no cumple la ley de frecuencias de Raunkjaer que se expresa por $\mathrm{I}>\mathrm{II}>\mathrm{III}=$ $\mathrm{IV}<\mathrm{V}$ para un bosque primario o clímax que no ha sido alterado.

La distribución de frecuencias del diámetro a la altura del pecho-DAP (figura 4) muestra una población dominante (moda) en torno a los $5 \mathrm{~cm}$ de DAP, 
FIGURA 3

DISTRIBUCIÓN DE LAS FRECUENCIAS (\%) POR CLASES DE PRESENCIA (I A V)

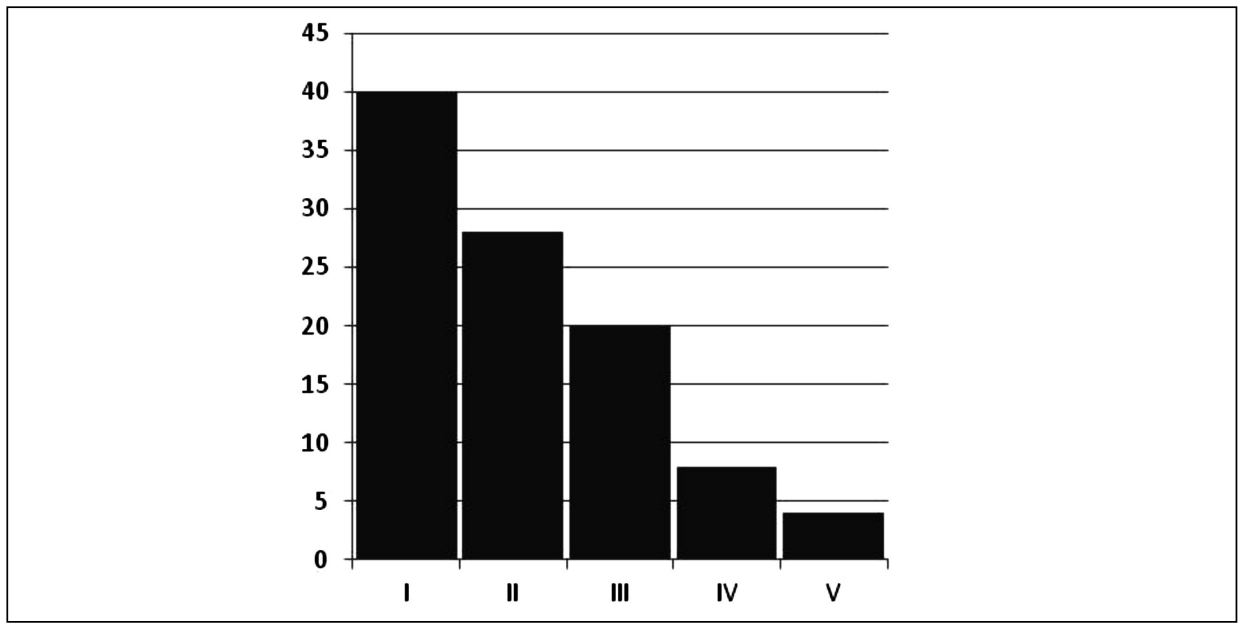

Fuente: elaboración propia.

FIGURA 4

DISTRIBUCIÓN DE FRECUENCIAS (EJE Y: \%) DE DAP (EJE X: EN CENTÍMETROS) DE LOS SABINOS

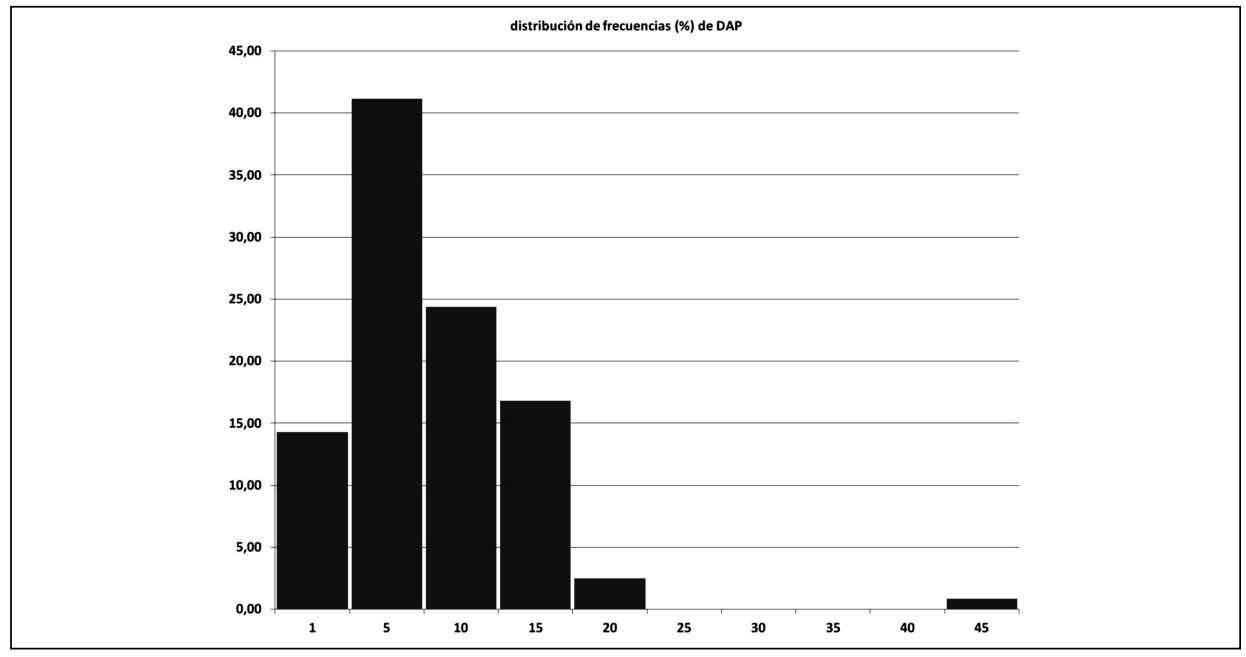

Fuente: elaboración propia.

Estudios Geográficos, Vol. LXXIV, 274, pp. 89-114, enero-junio 2013 ISSN: 0014-1496, eISSN: 1988-8546, doi: 10.3989/estgeogr.201304 
seguida de elementos entre 10 y $15 \mathrm{~cm}$, y de forma aislada algunos ejemplares de $45 \mathrm{~cm}$. Existe un hiato importante entre los elementos de hasta $20 \mathrm{~cm}$ de DAP y los de $45 \mathrm{~cm}$, que está relacionado con el manejo de este bosque anterior a la constitución de la Reserva, ya que, como indica Cobo (2008) «la madera de sabino era muy utilizada para la construcción de almadrabas y para las vigas de las chozas de Doñana por su resistencia a la humedad y a los insectos» (p. 55).

\section{Análisis de diversidad de las parcelas}

Los índices de diversidad (tabla 2) muestran en el índice Shannon valores entre 0,3 y 2,2, con una media de 1,66. Los datos ofrecen en general una equidad media-alta con alta dominancia, por debajo de 0,5 en el índice de BergerParker. Los valores altos de Simpson entre 0,70 y 0,86, expresan la dominancia del Juniperus phoenicea subsp. turbinata (Guss.) Nyman, frente a otras especies presentes en las parcelas CHTv y NVTv.

El índice de Sorensen (índice $\beta$ (tabla 3 ) muestra una relación de alta de similitud entre las parcelas CHTv 14, 15 y 16 (sabinar al norte de la laguna Charco del Toro), CHTV 27, 28, 29 y 30 (sabinar al sur de la laguna Charco del Toro), y NVT 18 y 19 al suroeste de Navazo del Toro.

El cálculo del índice $\beta$ en base al análisis de similitud de Morisita (tabla 4), que considera la abundancia de especies en el análisis multivariado, refuerza la relación de las parcelas CHTvl4, 15 y 16, a las que se asocia CHTv13, 17, 18 y 28 y deja aislada CHTv27 que se relaciona solamente con CHTv18. Otros dos grupos identificados son el conformado por CHTv29 y 30, y el grupo de NVTv18 y 19. La parcela NVT-18 aparece con este análisis, relacionada con CHTv15, 16, 17 y 28. El análisis cluster por el método de Morisita (figura 5) de las 12 parcelas establece 5 grupos claros.

TABLA 2

ÍNDICES $\alpha$ DE LAS PARCELAS DE SABINOS EN EL ÁREA DE ESTUDIO DE LA RBD

\begin{tabular}{|l|r|r|r|r|r|r|r|r|r|r|r|r|}
\hline & CHTv13 & CHTv14 & CHTv15 & CHTv16 & CHTv17 & CHTv18 & CHTv27 & CHTv28 & CHTv29 & CHTv30 & NVTv18 & NVTv19 \\
\hline Especies & 2 & 9 & 7 & 10 & 3 & 10 & 13 & 6 & 5 & 8 & 11 & 15 \\
\hline Individuos & 20 & 33 & 33 & 40 & 17 & 38 & 54 & 43 & 69 & 41 & 96 & 135 \\
\hline Shannon_H & 0.33 & 1.80 & 1.48 & 1.78 & 0.80 & 1.91 & 2.02 & 1.29 & 1.42 & 1.83 & 1.81 & 2.20 \\
\hline Simpson_1-D & 0.18 & 0.79 & 0.70 & 0.76 & 0.46 & 0.81 & 0.82 & 0.62 & 0.74 & 0.81 & 0.78 & 0.86 \\
\hline Equitability_J & 0.47 & 0.82 & 0.76 & 0.77 & 0.73 & 0.83 & 0.79 & 0.72 & 0.88 & 0.88 & 0.75 & 0.81 \\
\hline Berger-Parker & 0.90 & 0.30 & 0.45 & 0.43 & 0.71 & 0.29 & 0.31 & 0.58 & 0.32 & 0.32 & 0.35 & 0.21 \\
\hline
\end{tabular}

Fuente: elaboración propia. 
TABLA 3

ÍNDICE $\beta$ : RESULTADO DEL ANÁLISIS DE SIMILITUD DE SORENSEN. LOS VALORES POR ENCIMA DE 0.6 ESTÁN MARCADOS EN NEGRITA

\begin{tabular}{|c|c|c|c|c|c|c|c|c|c|c|c|c|}
\hline & CHTv_13 & CHTv_14 & CHTv_15 & CHTv_16 & CHTv_17 & CHTv_18 & CHTv_27 & CHTv_28 & CHTv_29 & CHTv_30 & NVTv_18 & NVTv_19 \\
\hline CHTv_13 & 1.0 & 0.2 & 0.2 & 0.2 & 0.4 & 0.3 & 0.1 & 0.3 & 0.3 & 0.2 & 0.2 & 0.1 \\
\hline CHTv_14 & 0.2 & 1.0 & 0.8 & 0.8 & 0.3 & 0.4 & 0.5 & 0.4 & 0.3 & 0.5 & 0.4 & 0.3 \\
\hline CHTv_15 & 0.2 & 0.8 & 1.0 & 0.7 & 0.4 & 0.5 & 0.5 & 0.5 & 0.3 & 0.3 & 0.6 & 0.4 \\
\hline CHTv_16 & 0.2 & 0.8 & 0.7 & 1.0 & 0.5 & 0.6 & 0.5 & 0.4 & 0.3 & 0.4 & 0.4 & 0.5 \\
\hline CHTv_17 & 0.4 & 0.3 & 0.4 & 0.5 & 1.0 & 0.5 & 0.4 & 0.4 & 0.3 & 0.2 & 0.3 & 0.3 \\
\hline CHTv_18 & 0.3 & 0.4 & 0.5 & 0.6 & 0.5 & 1.0 & 0.5 & 0.3 & 0.4 & 0.3 & 0.4 & 0.5 \\
\hline CHTv_27 & 0.1 & 0.5 & 0.5 & 0.5 & 0.4 & 0.5 & 1.0 & 0.7 & 0.6 & 0.5 & 0.4 & 0.5 \\
\hline CHTv_28 & 0.3 & 0.4 & 0.5 & 0.4 & 0.4 & 0.3 & 0.7 & 1.0 & 0.5 & 0.4 & 0.4 & 0.4 \\
\hline CHTv_29 & 0.3 & 0.3 & 0.3 & 0.3 & 0.3 & 0.4 & 0.6 & 0.5 & 1.0 & 0.6 & 0.4 & 0.4 \\
\hline CHTv_30 & 0.2 & 0.5 & 0.3 & 0.4 & 0.2 & 0.3 & 0.5 & 0.4 & 0.6 & 1.0 & 0.4 & 0.4 \\
\hline NVTv_18 & 0.2 & 0.4 & 0.6 & 0.4 & 0.3 & 0.4 & 0.4 & 0.4 & 0.4 & 0.4 & 1.0 & 0.7 \\
\hline NVTv_19 & 0.1 & 0.3 & 0.4 & 0.5 & 0.3 & 0.5 & 0.5 & 0.4 & 0.4 & 0.4 & 0.7 & 1.0 \\
\hline
\end{tabular}

Fuente: elaboración propia.

TABLA 4

ÍNDICE $\beta$ : RESULTADO DEL ANÁLISIS DE SIMILITUD DE MORISITA. LOS VALORES POR ENCIMA DE 0.6 ESTÁN MARCADOS EN NEGRITA

CHTv_13 CHTv_14 CHTv_15 CHTv_16 CHTv_17 CHTv_18 CHTv_27 CHTv_28 CHTv_29 CHTv_30 NVTv_18 NVTv_19

\begin{tabular}{|c|c|c|c|c|c|c|c|c|c|c|c|c|}
\hline CHTv_13 & 1.0 & 0.5 & 0.7 & 0.7 & 0.9 & 0.5 & 0.4 & 0.9 & 0.5 & 0.2 & 0.6 & 0.1 \\
\hline CHTv_14 & 0.5 & 1.0 & 0.9 & 0.9 & 0.6 & 0.6 & 0.5 & 0.6 & 0.6 & 0.3 & 0.6 & 0.3 \\
\hline CHTv_15 & 0.7 & 0.9 & 1.0 & 1.0 & 0.8 & 0.6 & 0.6 & 0.8 & 0.5 & 0.2 & 0.7 & 0.2 \\
\hline CHTv_16 & 0.7 & 0.9 & 1.0 & 1.0 & 0.8 & 0.7 & 0.6 & 0.8 & 0.5 & 0.3 & 0.7 & 0.3 \\
\hline CHTv_17 & 0.9 & 0.6 & 0.8 & 0.8 & 1.0 & 0.7 & 0.5 & 0.9 & 0.5 & 0.2 & 0.7 & 0.2 \\
\hline CHTv_18 & 0.5 & 0.6 & 0.6 & 0.7 & 0.7 & 1.0 & 0.8 & 0.6 & 0.4 & 0.2 & 0.6 & 0.4 \\
\hline CHTv_27 & 0.4 & 0.5 & 0.6 & 0.6 & 0.5 & 0.8 & 1.0 & 0.6 & 0.4 & 0.2 & 0.5 & 0.5 \\
\hline CHTv_28 & 0.9 & 0.6 & 0.8 & 0.8 & 0.9 & 0.6 & 0.6 & 1.0 & 0.6 & 0.3 & 0.8 & 0.3 \\
\hline CHTv_29 & 0.5 & 0.6 & 0.5 & 0.5 & 0.5 & 0.4 & 0.4 & 0.6 & 1.0 & 0.8 & 0.5 & 0.2 \\
\hline CHTv_30 & 0.2 & 0.3 & 0.2 & 0.3 & 0.2 & 0.2 & 0.2 & 0.3 & 0.8 & 1.0 & 0.2 & 0.2 \\
\hline NVTv_18 & 0.6 & 0.6 & 0.7 & 0.7 & 0.7 & 0.6 & 0.5 & 0.8 & 0.5 & 0.2 & 1.0 & 0.7 \\
\hline NVTv_19 & 0.1 & 0.3 & 0.2 & 0.3 & 0.2 & 0.4 & 0.5 & 0.3 & 0.2 & 0.2 & 0.7 & 1.0 \\
\hline
\end{tabular}

Fuente: elaboración propia. 
FIGURA 5

OBTENCIÓN DE 5 GRUPOS EN BASE AL ANÁLISIS CLUSTER SEGÚN EL MÉTODO DE SIMILITUD DE MORISITA OBTENIDO CON LA APLICACIÓN DEL SOFTWARE PAST

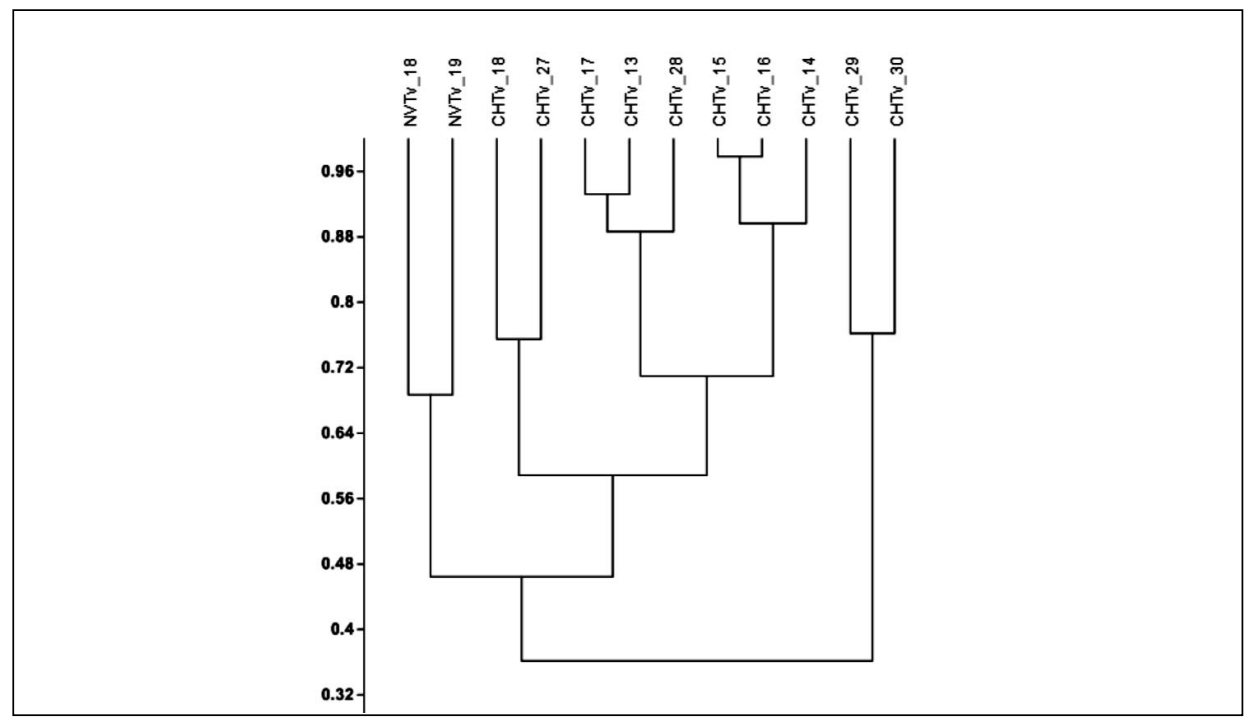

Fuente: elaboración propia.

\section{Características estructurales de la formación vegetal}

El patrón de distribución de alturas expresado en el histograma de la figura 6 muestra un bosque de sabinos de una altura media de 3,5 $\mathrm{m}$ con elementos localizados y dispersos emergentes de hasta 6 y $7 \mathrm{~m}$ de altura. Al igual que en el histograma de DAP se observa un hiato entre los 5 y $6 \mathrm{~m}$, apareciendo una moda entre 6 y 5,5 metros, y algunos ejemplares con valores entre 7 y $8 \mathrm{~m}$. Otro dato importante es el bajo número de ejemplares por debajo de $1,5 \mathrm{~m}$, que informa de un bosque con una tasa de renovación muy baja en la actualidad, encontrándose la mayoría de los individuos entre 2 y 4,5 m.

El Indice de Valor de importancia (IVI) (Curtis, 1951) sintetiza los valores de presencia, abundancia y cobertura de las especies en las 12 parcelas de la muestra. El valor más alto es el de Juniperus phoenicea subsp. turbinata (Guss.) Nyman con una media de 54,86 (desviación estándar 18), con un máximo de 85 (bosque cerrado) y un mínimo de 28 (bosque muy abierto), por lo que po- 
FIGURA 6

DISTRIBUCIÓN DE FRECUENCIAS (EJE Y: \%) DE LAS ALTURAS

(EJE X: EN METROS) DE LOS SABINOS

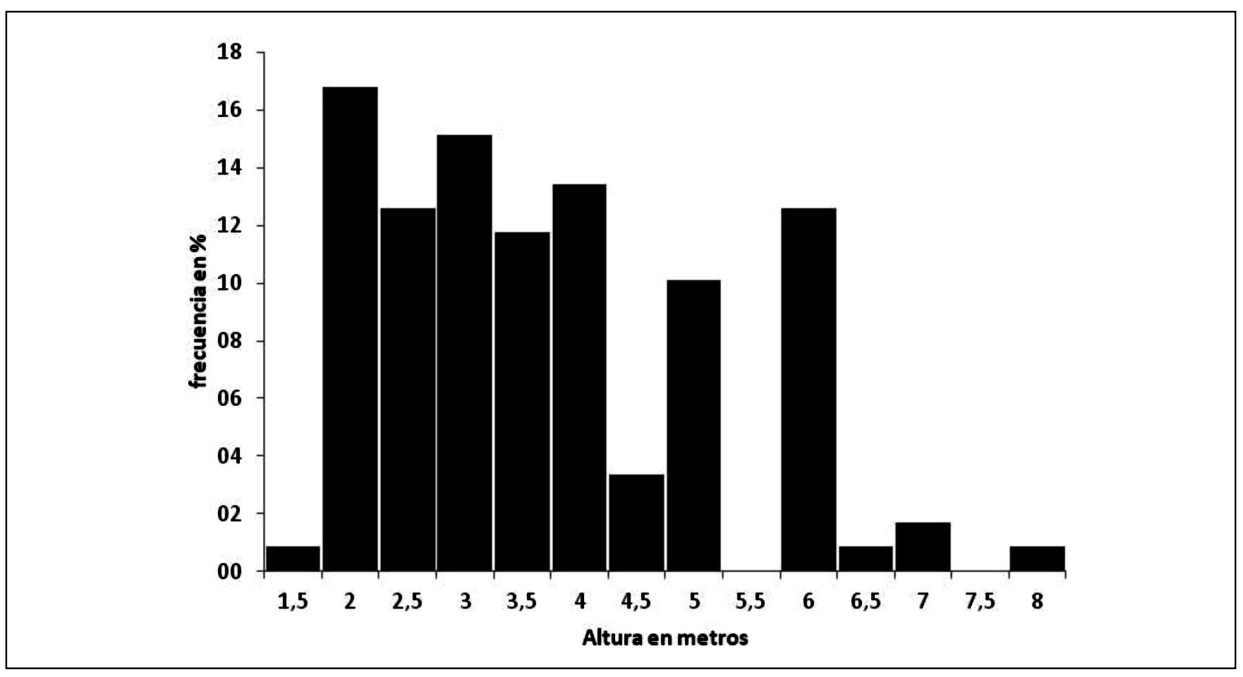

Fuente: elaboración propia.

demos hablar de una formación arbórea abierta de sabinar a la que acompaña un matorral de Halimium halimifolium (L.) Willk. in Willk. \& Lange y Rosmarinus officinalis L., y localmente, según las características de disponibilidad hídrica subsuperficial con Erica scoparia L., o sin disponibilidad hídrica con Lavandula pedunculata (Mill.) Cav., Stauracanthus genistoides (Brot.) Samp., Pinus pinea L., Halimium calycinum (L.) K. Koch, y Cistus libanotis L. (tabla 5).

A partir de los datos expuestos y de análisis cluster realizado, hemos obtenido 5 grupos homogéneos de los sabinares en la RBD:

- Grupo 1: NVTv18 y 19. Constituye el sabinar del Ojillo al suroeste de Navazo del Toro, una formación abierta de Juniperus phoenicea subsp. turbinata (Guss.) Nyman (IVI medio 35), localizada en el AMEs, con una altura media de 3-5 $\mathrm{m}$ y una cobertura semicerrada en el estrato arbustivo y subarbustivo (figura 7). La riqueza de especies presenta, como elemento diferenciador de los otros grupos florísticos, la abundancia de individuos de Cistus libanotis L., y Lavandula pedunculata (Mill.) Cav., a los que acompañan Halimium halimifolium (L.) Willk. in Willk. \& Lange, Halimium calycinum (L.) K. Koch y Rosmarinus officinalis L., que 


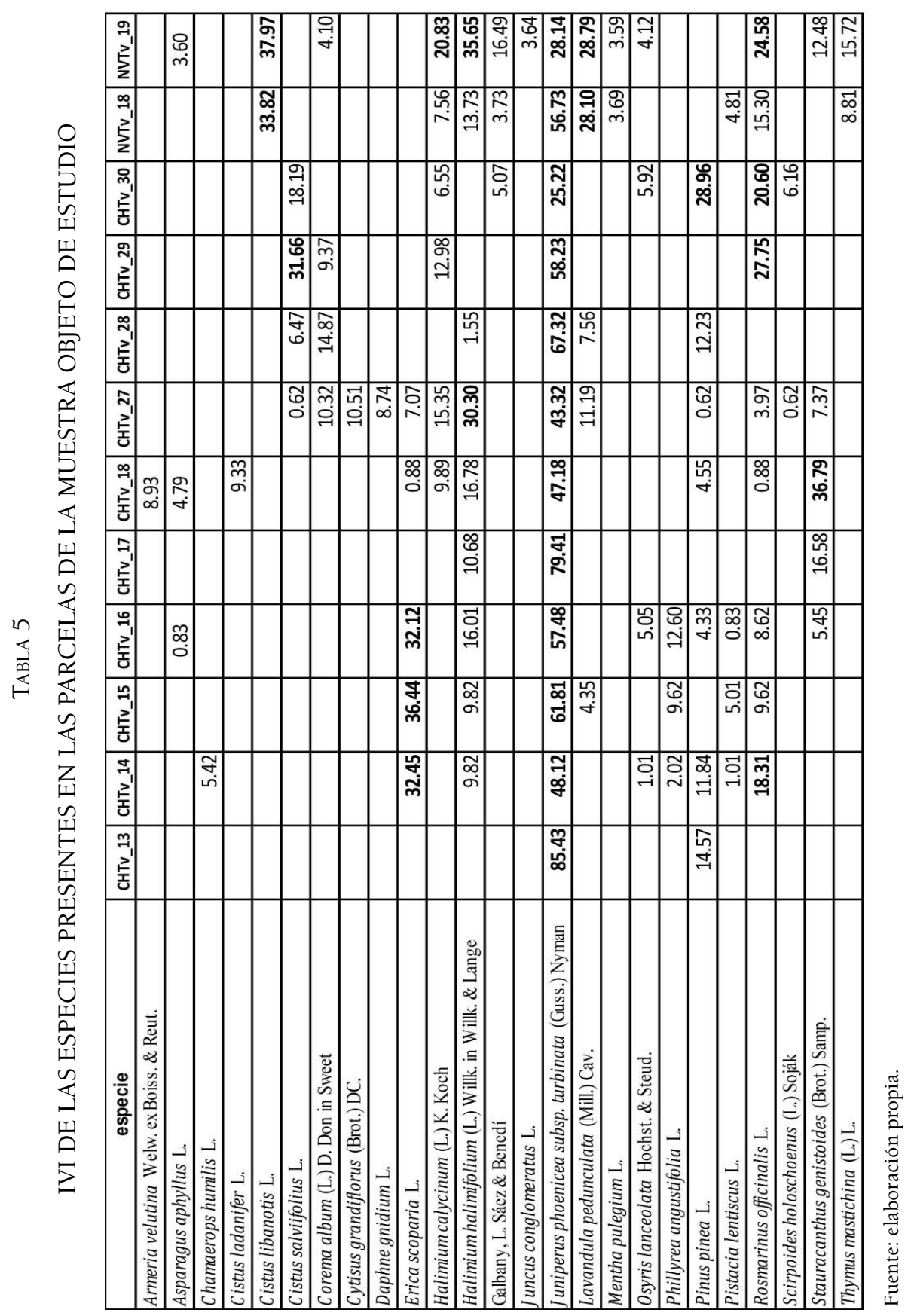

Estudios Geográficos, Vol. LXXIV, 274, pp. 89-114, enero-junio 2013 ISSN: 0014-1496, eISSN: 1988-8546, doi: 10.3989/estgeogr.201304 
PARCELA NVTV19. DEREC TIPO CORRESPONDIENTE AL GRUPO 1

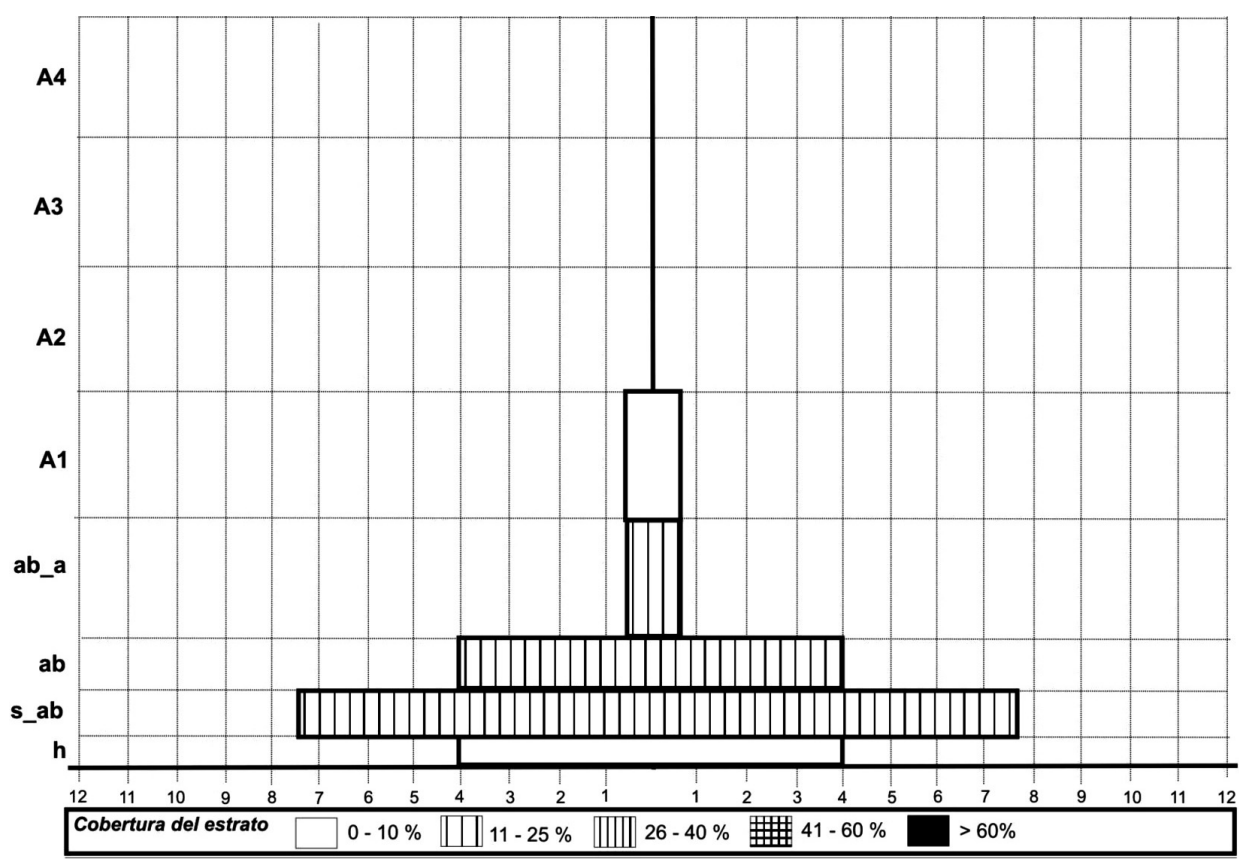

Fuente: elaboración propia.

aparecen agrupados en el transecto en los espacios abiertos (figura 8). El índice de Shannon se sitúa en torno a 2, con alta dominancia (Simpson en torno a 0,8), y baja equitabilidad con Berger-Parker entre 0,21 y 0,35. Esta formación de sabinar sobre dorsos y superficies dunares arrasados presenta horizontes con alta conductividad superficial y subsuperficial y ausencia de rasgos hidromorfos, lo que confiere a la misma una alta característica de xericidad. Dada la ausencia de pinares en este grupo y su elevada diversidad de Shannon con respecto a los otros grupos, así como por su situación no ecotónica, se considerará la parcela NVTv 19 de este grupo como DEREC tipo de referencia geobotánica.

- Grupo 2: CHTv18 y 27. Se localiza al norte y sur, respectivamente, de la laguna de Charco del Toro sobre el AMEs, en tránsito a cubetas lagunares no funcionales. Es una formación cerrada de Juniperus phoenicea subsp. turbinata (Guss.) Nyman con IVI medio 55,8, altura media baja en 
FIGURA 8

DIAGRAMA DE BURBUJAS QUE MUESTRA LA COBERTURA HORIZONTAL DE LA PARCELA NVTV19

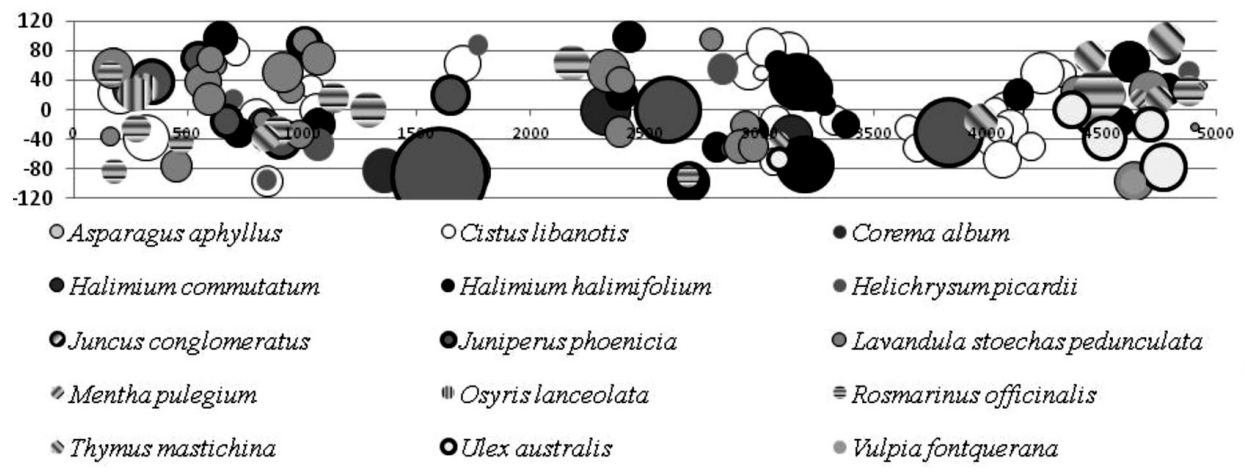

Fuente: elaboración propia.

torno a 3-5 m, con los estratos arbustivo (ab) y arbóreo bajo (Al) muy abiertos (11-25\%) (figura 9a). Presenta una cobertura horizontal muy abierta en el transecto con los individuos de Juniperus muy separados (figura 10). Tiene como carácter diferenciador la presencia de Halimium halimifolium (L.) Willk. in Willk. \& Lange y Stauracanthus genistoides (Brot.) Samp. y como elemento característico Corema album (L.) D. Don in Sweet. El índice de Shannon tiene un valor entre 1,91 y 2, con media a alta dominancia (Simpson 0,81 a 0,82 ) y baja a media equitabilidad (0,29 a 0,31 con Berger-Parker). Las parcelas de este grupo constituyen una transición con pinar desde los dorsos dunares del AMEs hacia depresiones interdunares y cubetas lagunares sin funcionamiento hídrico subsuperficial actual. Solo en los años muy húmedos (superior a $700 \mathrm{~mm}$ ) la remontada del freático inunda temporalmente las depresiones y cubetas, permaneciendo los arenales dunares con alta conductividad en sus horizontes.

- Grupo 3: CHTv29 y 30. Situados al sur de la laguna de Charco del Toro en un transecto de transición entre el AMEs y las dunas activas con blowout y semiestabilizadas. Constituye una formación cerrada de Juniperus phoenicea subsp. turbinata (Guss.) Nyman (IVI medio 41,5) de altura media baja en torno a 3-4 m con el estrato arbóreo bajo (Al) muy abierto (11-25\%) (figura 9b). En el conjunto el transecto presenta una 
FIGURA 9

\section{DERECS TIPO DE LOS GRUPOS 2 A 5 Y SU DINÁMICA TOMANDO COMO REFERENCIA NVTV-19 DEL GRUPO 1}

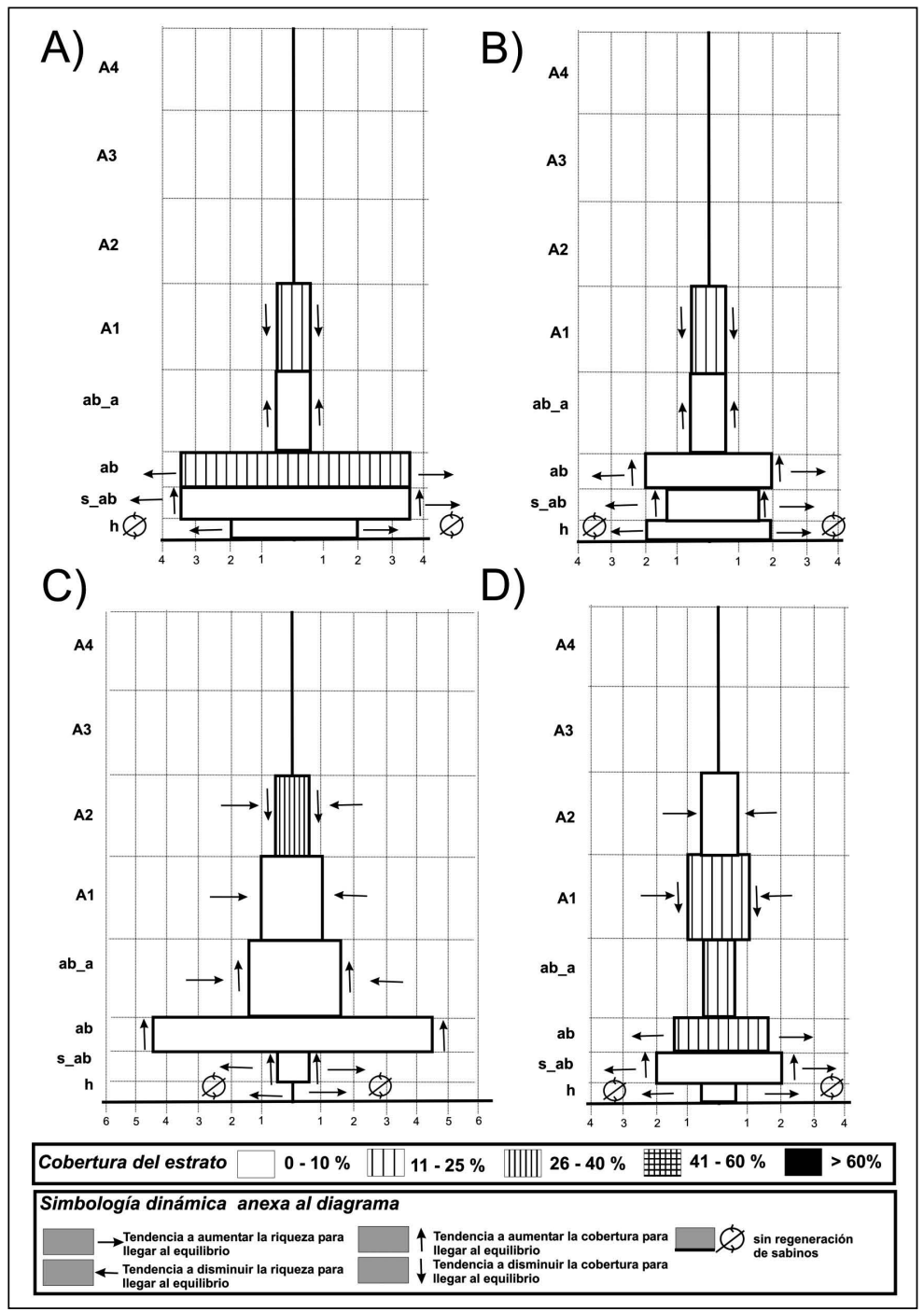

Nota: a) tipo grupo 2: CHTv-18; b) tipo grupo 3: CHTv-29; c) tipo grupo 4: CHTv-16; d) tipo grupo 5: CHTv-28.

Fuente: elaboración propia. 
FIGURA 10

DIAGRAMA DE BURBUJAS QUE MUESTRA LA COBERTURA HORIZONTAL DE LA PARCELA CHTV18

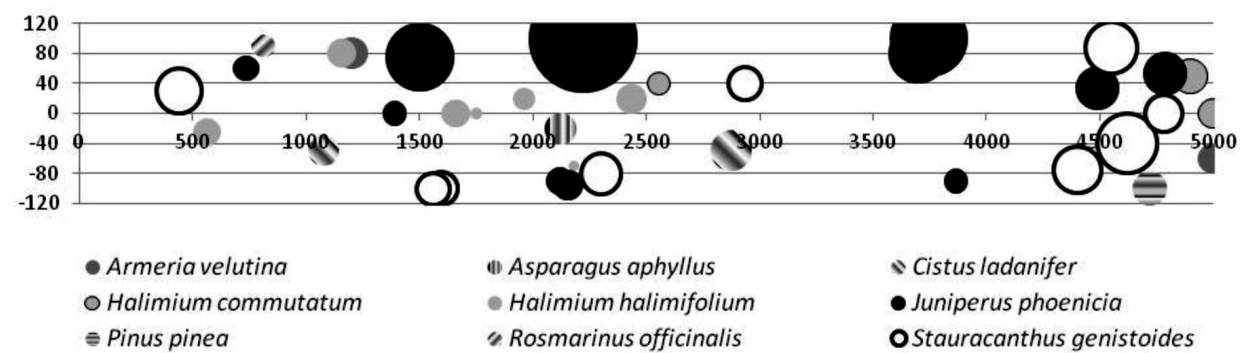

Fuente: elaboración propia.

cobertura abierta en la que Juniperus queda en pequeños rodales (figura 11) con Rosmarinus officinalis L., acompañado de Pinus pinea L. y Cistus salviifolius L., que es más dominante conforme el transecto se acerca a las dunas activas, y como elemento característico Corema album (L.) D. Don in Sweet. El índice de Shannon está entre 1,42 y 1,83, con media a alta dominancia (Simpson 0,74 a 0,81 ) y baja a media equitabilidad (0,32 a 0,35 con Berger-Parker). En ambas parcelas las características de los horizontes de las formaciones dunares son de alta conductividad sin rasgos hidromorfos, con ausencia total de encharcamientos.

FIGURA 11

DIAGRAMA DE BURBUJAS QUE MUESTRA LA COBERTURA HORIZONTAL DE LA PARCELA CHTV29

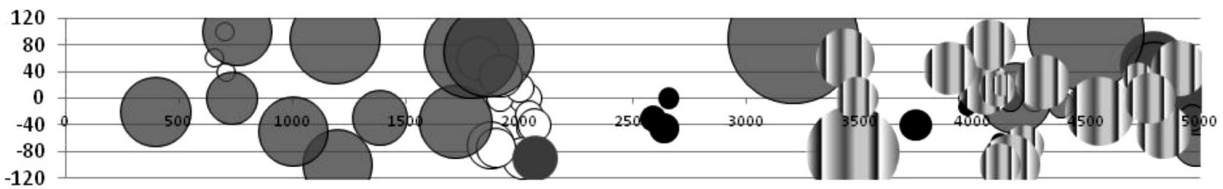

O Cistus salviifolius $\bullet$ Corema album $\bullet$ Halimium commutatum O Juniperus phoenicia 1 Rosmarinus officinalis

Fuente: elaboración propia. 
- Grupo 4: CHTv14, 15, 16. Emplazado al norte de la laguna de Charco del Toro, sobre el AMEs y con circulación hídrica subsuperficial transversal al transecto de norte a sur. Constituye una formación arbórea cerrada con IVI medio de 50 de Juniperus phoenicea subsp. turbinata (Guss.) Nyman. La altura media es de 5 a 10 metros, abierta en los estratos inferiores y cerrada (28-40\%) en el superior (A2) (figura 9c). Con una disposición concentrada de los individuos de Juniperus phoenicea subsp. turbinata (Guss.) Nyman, ocupando los espacios abiertos entre ellos Erica scoparia L. (figura 12), que es el elemento florístico dominante, acompañada localmente por Pinus pinea L., Phillyrea angustifolia L., Pistacia lentiscus L, Rosmarinus officinalis L. y Halimium halimifolium (L.) Willk. in Willk. \& Lange. y destacando la ausencia de Halimium calycinum (L.) K. Koch. Los ejemplares de Juniperus phoenicea subsp. turbinata (Guss.) Nyman) de $6 \mathrm{~cm}$ de DAP y Erica scoparia L. con DAP mayor de $3 \mathrm{~cm}$, muestran abundantes trazas de quemas anteriores a la declaración de la RBD. Los valores del índice de Shannon alcanza 1,8 , con alta dominancia (Simpson 0,7 a 0,79 ) y equitabilidad media a baja $(0,3$ a 0,45$)$. El emplazamiento de estas parcelas en el AMEs presenta unas formaciones dunares con alta conductividad, que sin embargo a lo largo del transecto recoge antiguas depresiones en transición a los humedales del AMEh, donde se localiza Erica scoparia L. respondiendo a un funcionamiento hídrico mesogénico (Borja, 2002; Borja y Díaz del Olmo, 2008, 2009).

FIGURA 12

DIAGRAMA DE BURBUJAS QUE MUESTRA LA COBERTURA HORIZONTAL DE LA PARCELA CHTV16

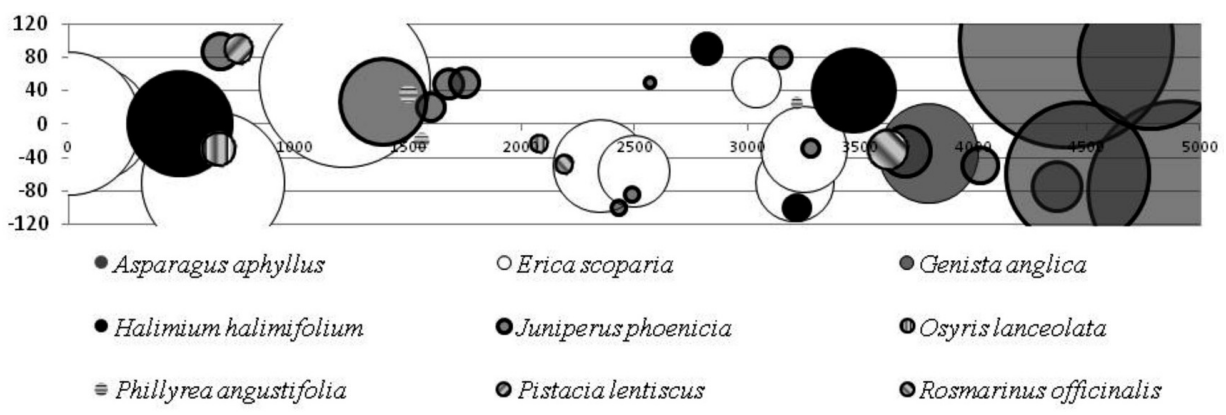

Fuente: elaboración propia. 
- Grupo 5: CHTv13, 17 y 28. Localizado al norte y sur de la laguna de Charco del Toro sobre el AMEs con horizontes de alta conductividad. La altura media de la formación vegetal es $4,5 \mathrm{~m}$, con presencia de Pinus pinea L. y Halimium halimifolium (L.) Willk. in Willk. \& Lange. El estrato arbóreo bajo (Al) y el arbustivo (ab) presentan una cobertura muy abierta (11-25\%) (figura 9d) que en el transecto muestra a Juniperus muy distanciados dejando espacios abiertos (figura 13). Alcanza valores altos en el índice de Shannon (2 nats), y dominancia muy variable en las tres parcelas: muy baja en CHTvl3, media en CHTvl7 y alta en CHTv18. La equitabilidad es media a alta $(0,58$ a 0,90$)$.

FIGURA 13

DIAGRAMA DE BURBUJAS QUE MUESTRA LA COBERTURA HORIZONTAL DE LA PARCELA CHTV28

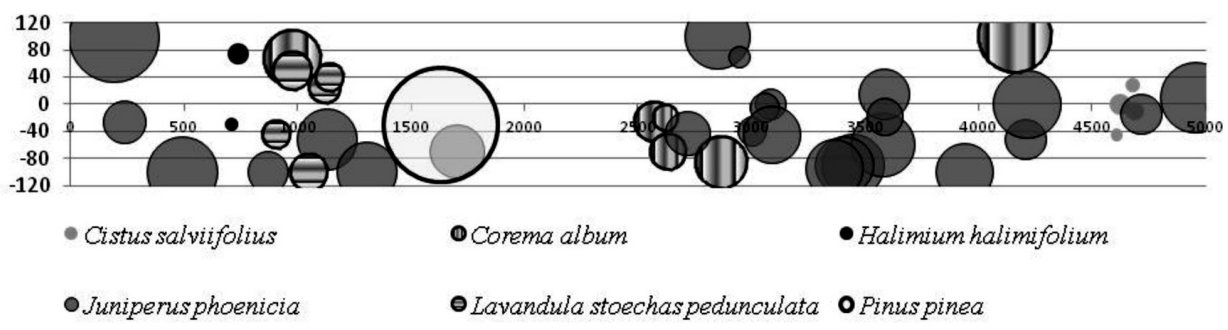

Fuente: elaboración propia.

\section{Caracterización dinámica de la formación de sabinos}

Como se ha indicado los resultados de las parcelas correspondientes al Grupo 1 constituyen la formación tipo de los sabinares de la RBD, dada su adaptación a las condiciones edafoxerófilas del AMEs.

Tomando dicha referencia se han establecido los DEREC de los 4 grupos restantes que muestran por estratos la dinámica en riqueza de especies y cobertura, teniendo en cuenta la localización detallada y los factores de presión ambiental que sobre las parcelas se establece. Los grupos 2 y 3 (figuras 9a y 9b) presentan una similitud de estructura en los estratos arbóreos bajo (Al) y arbustivo alto (ab_a) con una dinámica inversa en cada uno de ellos a dismi- 
nuir o aumentar la cobertura respectivamente; por su parte el estrato herbáceo es igualmente similar con tendencia aumentar su riqueza, sin detectar en la actualidad regeneración de sabinos. La mayor diferenciación se produce en los estratos arbustivo (ab) y subarbustivo (s_ab) donde la riqueza de especies es mayor en el grupo 2 que en el 3, e igualmente la cobertura del estrato arbustivo del grupo 2 respecto del 3 . No obstante en ambos estratos y grupos deben aumentar la riqueza y aunque menos la cobertura. Los rasgos de similitud dinámica establecidos entre los grupos 2 y 3 confluyen en el carácter de ecotono de los mismos, con independencia de las particularidades de ambos grupos: el grupo 2 hacia depresiones interdunares y cubetas lagunares con brezales, y el 3 hacia complejos dunares con morfogénesis eólica activa o dunas fitoestabilizadas y presencia de Pinus pinea L. de bajo porte $(<5 \mathrm{~m})$.

Por su parte los DEREC de los grupos 4 y 5 (figuras 9 c y $9 d$ ), que ofrecen las mayores alturas de las formación vegetal derivada de la presencia de pinos (entre 5 a $10 \mathrm{~m}$ ), reflejan dos condiciones de estructura y cobertura muy diferentes entre sí en sus estratos, siendo así que sin embargo responden al mismo contexto hidrogeomorfológico del AMEs. El estrato arbóreo A2 presenta una cobertura semicerrada en el grupo 4 y abierta en el 5; invirtiéndose la cobertura respectivamente en los estratos arbóreo (A1), arbustivo alto (ab_a) y arbustivo (ab) inferiores. El elemento diferencial en ellos la mayor cobertura ligada a la presencia de Juniperus y Corema album (L.) D. Don in Sweet en el Grupo 5. En los estratos subarbustivo y herbáceo el número de especies se ve muy disminuido en el grupo 4 y con una tendencia a aumentar la riqueza. En el contexto de ambos grupos se manifiesta una tendencia general hacia la disminución de la riqueza de especies, frente a los estratos inferiores que deben tender a aumentar.

\section{DISCUSIÓN}

López (2012) identifica a través del análisis multivariante K-mean 23 grupos que se correspondieron con 15 asociaciones diferentes. Con este análisis clasifica 321 inventarios, aproximadamente un $45 \%$ del total de los realizados. De estos 23 grupos, 2 presentan a los sabinos como dominantes, el 10 y el 16. En el primero identificó tres comunidades fitosociológicas: Osyrio quadripartitae-Juniperetum turbinatae, Osyrio quadripartitae-Juniperetum turbinatae galietosum minutuli y Rhamno oleoidis-Juniperetum macrocarpae. En el grupo 16 es semejante al 10, pero se diferencia por el cortejo florístico anual, marcado por la diferencia en las precipitaciones entre los años de muestreo. Según estos 
resultados identifica una Serie edafoxerófila del sabinar que relaciona con la capa freática, alejada de la superficie. En las áreas más elevadas, donde la capa freática está muy alejada de la superficie, aparece un cortejo de especies de matorral xerófito en el que abunda Rosmarinus officinalis L., Cistus libanotis L., Halimium halimifolium (L.) Willk. in Willk. \& Lange. y Halimium calycinum (L.) K. Koch entre otras. La etapa previa al sabinar la representa, según López (2009), el monte blanco (Halimio halimifolii-Stauracanthetum genistoidis).

Las resultados de las parcelas presentadas en éste artículo refuerzan el carácter edafoxerófilo del sabinar. Sin embargo los datos obtenidos por los transectos muestran una estrecha correlación de las formaciones del sabinar con las unidades geomorfológicas de mantos eólicos: AMEs como dominante y tránsito al AMEh. El primero de ellos presenta en las arenas dunares siempre una alta conductividad, lo que se refleja en una constante xericidad de los horizontes. Incluso cuando en ciclos pluviométricos muy húmedos, las depresiones interdunares, topografías bajas de blowout y antiguas cubetas lagunares, puedan sostener durante algunos días o semanas láminas de agua de potencia centimétrica. Sobre estas unidades se han implantado los bosques de nave, de los cuales la formación tipo actual presenta los rasgos estructurales y florísticos del Grupo 1. Por su parte en el AMEh se han detectado dos tipos de funcionamiento hídrico en las cubetas lagunares: el funcionamiento mesogénico o subsuperficial, que afecta igualmente a algunas superficies de enlace entre el dorso dunar y la cubeta laguna, en el que los sabinares se adecuan a los dorsos con alta conductividad hidráulica; y el funcionamiento hipogénico derivado la fluctuación del freático, donde no hay presencia de sabinos. En este manto, la existencia de humedales, hace que la proliferación de sabinos se vea condicionada por la situación de ecotonos, colonizando con preferencia las formaciones con alta conductividad hidráulica y alejándose de los ámbitos con funcionamiento hídrico más permanente. A nivel de variedad de especies y de estructura de la vegetación hay que resaltar la existencia de blowout colonizados con Corema album (L.) dispersa, lo que se asocia a una intensificación de la morfogénesis eólica litoral.

La dinámica vegetal que ofrecen los grupos, tomando como referencia geobotánica el Sabinar de El Ojillo (Grupo 1), es desigual, aunque de forma general todos tienden en sus estratos a aumentar la riqueza de especies. Los grupos 2,3 y 4 son los que poseen un IVI para el Juniperus similar, con valores por debajo de $60 \%$. Sólo el Grupo 5 manifiesta un IVI por encima del $60 \%$, alcanzando el 85\%. Estas condiciones subrayan la importancia del factor condicionante de ecotono (Grupos 2 y 3) frente al de un mayor manejo histórico del bosque de sabinos (Grupos 4 y 5 ). 


\section{CONCLUSIONES}

El análisis de los sabinares de la RBD mediante el MIFC establece parámetros de caracterización cuantificada que permite la comparación entre diferentes agrupaciones y rodales. La base comparativa de la estructura, dinámica y riqueza vegetal se establece a partir de los DEREC, el IVI y los índices de diversidad y, a través del conjunto de 12 parcelas encadenadas en 5 transectos de $50 \mathrm{~m}$ (600 m de longitud, $1.200 \mathrm{~m}^{2}$ de superficie).

Los sabinares de la RBD constituyen un bosque de naves bien conocido desde el punto de vista paisajístico y fitosociológico. Se trata una formación con estructura vertical con un estrato subarbustivo (s_ab) rico en especies de fanerófitos y caméfitos, que en el nivel arbustivo (ab) se reduce hasta alcanzar unos estratos arbustivo alto (a_ab) y arbóreo bajo (Al) monoespecíficos. La mayor cobertura recae en los estratos arbustivos con valores entre 26 al $40 \%$. El conjunto presenta un índice de Shannon de 2 nats, siendo los valores más bajos 0,33 nats y los mayores 2,20 nats. La dominancia expresada a través de índice de Simpson presenta valores elevados oscilando entre 0.60 y 0.80 , que denota la dominancia de Juniperus phoenicea subsp. turbinata (Guss.) Nyman, corraborada a través de un alto IVI para esta especie (un valor medio de 55\%).

Desde el punto de vista geobotánico, si bien la xericidad es el factor relevante para comprender la distribución del sabinar, su ubicación en el AMEs se relaciona más con la ausencia de circulación hídrica subsuperficial, que con la profundidad del freático (aguas subterráneas) respecto de la superficie morfotopográfica deprimida del manto eólico. En tal sentido es particularmente determinante el carácter de la alta conductividad de las arenas y horizontes edáficos y de alteración sobre los que se distribuye el sabinar. El bosque de naves del entorno de Charco del Toro está condicionado por la transición entre las Dunas Semiestables, el AMEs y el AMEh, así como los factores de presión antrópica anterior a la declaración de la RBD.

En los sabinos de la RBD hemos identificado 5 grupos con estructura y composición florística: 1 formación cerrada de sabinar (Grupo 2), y tres de sabinar-pinar (Grupos 3, 4 y 5) en Charco del Toro; y una quinta abierta sin pinar (sabinar de El Ojillo), más xérica que las anteriores, al suroeste de la Laguna de Navazo del Toro.

Fecha de recepción: 01/08/2012

Fecha de aceptación: 21/03/2013 


\section{BIBLIOGRAFÍA}

Allier, C. (1977): La végétation psammophile du litoral de la Reserve Biologique de Doñana. Monografía ICONA, 18. Madrid, Publicaciones del Ministerio de Agricultura Pesca y Alimentacion, pp. 131-157.

Allier, C., Bernáldez, F. G. y Ramírez-Díaz, L. (1974): Mapa Ecológico de la Reserva Biológica de Doñana 1: 10.000. Monografías. Sevilla, Estación Biológica de Doñana, CSIC.

Asensi, A. y Díez Garretas, B. (1987): “Andalucía Occidental”, en M. Peinado y S. Rivas (coords.): La vegetación de España. Madrid, Universidad de Alcalá de Henares, pp. 197-230.

Bejarano, R. (1994): "Los paisajes vegetales de la costa onubense y su significación". Huelva en su Historia, 5, pp. 157-186.

Bejarano, R. (1997a): Vegetación y paisaje en la costa atlántica de Andalucía. Sevilla, Univ. Sevilla, 419 p.

Bejarano, R. (1997b): "El análisis de la vegetación como criterio de interpretación del paisaje (Andalucía atlántica)". Estudios Geográficos, LVIII/226, pp. 5-32.

Bejarano, R. (2004): "Tipología de los sabinares costeros según su estructura vertical y el grado de cobertura por especies", en J. M. Panareda et al. (eds.): Estudios en Biogeografía. Libro Homenaje a José Manuel Rubio Recio y Jesús García. Terrasa, Aster, pp. 59-74.

Bejarano, R., Cámara, R., Borja, C., Díaz del Olmo, F., Recio, J. M. y Borja, F. (2010): "Caracterización de los bosques de sabina (Juniperas turbinata Guss.) del entorno de la laguna Charco del Toro (Parque Nacional de Doñana, Huelva): aplicación de una nueva metodología para el estudio de la vegetación", en P. Giménez Font, J. A. Marco Molina, E. Matarredona Coll, A. Padilla Blanco y A. Sánchez Pardo (coords.): Biogeografía: una ciencia para la conservación del medio. VI Congreso Español de Biogeografía, Alicante. Alicante, Universidad de Alicante y Asociación de Geógrafos Españoles, pp. 25-34.

Blasco, F., Bellan, M. F. y Aizpuru, M. (1996): "A vegetation map of tropical continental Asia at scale 1:5 million”. Journal of Vegetation Science, 7, pp. 623-634.

Borja, C. y Díaz del Olmo, F. (1994): "Geomorfología del manto eólico litoral de El Abalario (Huelva)", en J. Arnáez-Vadillo, J. M. García Ruiz y A. Gómez Villar (eds.): Geomorfología en España. Logroño, SEG, pp. 327-338.

Borja, C., Díaz del Olmo, F. y Borja F. (2009): "Metodología hidrogeomorfológica y resultados en los humedales de Doñana", en J. A. López Geta y J. M. Fornés ( eds.): La geología e hidrogeología en la investigación de humedales. Serie Hidrogeología y aguas subterráneas, 28. Madrid, IGME, pp. 271-287.

Borja, C., Díaz del Olmo, F. y Borja, F. (2008): "Dinámica hidrogeomorfológica de la Laguna de Charco del Toro (Complejo Palustre del Manto Eólico Litoral de el Abalario-Doñana). Parque Nacional de Doñana, Huelva”. Territoris, 7-8, pp. 87-98. 
Borja, F. (1992): "Cuaternario Reciente, Holoceno y Períodos Históricos del SW de Andalucía. Paleogeografía de medios litorales y fluvio-litorales de los últimos 30.000 años". Tesis Doctoral. Universidad de Sevilla. Inédito, 520 pp.

Borja, F. (1997): "Dunas litorales de Huelva (SW de España). Tipología y secuencia Pleistoceno superior-Holoceno", en J. Rodríguez Vidal (ed.): Cuaternario Ibérico. Huelva, AEQUA. pp. 84-97.

Borja, F. y Díaz del Olmo, F. (1994): "Geomorfología del manto eólico litoral de El Abalario (Huelva)", en J. Arnáez-Vadillo, J. M. García Ruiz y A. Gómez Villar (eds.): Geomorfología en España. Logroño, SEG, pp. 327-338.

Borja, F. y Díaz del Olmo, F. (1996): "Manto eólico litoral (MEL) de El Abalario (Huelva, España): episodios morfogenéticos posteriores al 22.000 Bp", en A. Pérez Alberti, P. Martini, W. Chesworth y A. Martínez Cortizas (eds.): Dinámica y evolución de medios cuaternarios. Santiago de Compostela, Xunta de Galicia, pp. 375-390.

Cain, S. A. (1938): "The species-area curve". American Midland Naturalist Journal, 19, pp. 573-581.

Cámara, R. y Díaz del Olmo, F. (2013): "Muestreo en transecto de formaciones vegetales de fanerófitos y Caméfitos (I): Fundamentos metodológicos". Estudios Geográficos, 74/274, pp. 67-88.

Cobo, M. P. y Tijera, R. E. (2008): Etnografía de la Doñana sevillana. Sevilla. Mancomunidad de Desarrollo y Fomento del Aljarafe.

Costa, M., Morla, C. y Saiz Ollero, H. (1998): Los bosques ibéricos. Una interpretación geobotánica. Barcelona, Planeta, 598 pp.

Curtis J. T. y Mcintosh, R. P. (1951): "An upland forest continuum in the pariré-forest border region of Wisconsin". Ecology, 32, pp. 476-496.

Ferreras, C. y Arozena, M. E. (1987): Los bosques. Guía Física de España, 2. Madrid, Alianza Editorial, 594 pp.

Junta de Andalucía: "Memoria, y Mapa Ecológico de Doñana", E 1:40.000.

Kryshtofovich, A. N. (1929): "Evolution of the Tertiary Flora in Asia". New Phytologist, 28/4, pp. 303-312.

Kryshtofovich, A. N. (1935): “A Final Link between the Tertiary Floras of Asia and Europe (Contribution to the Age of the Arcto-Tertiary Floras of the Northern Holarctic)". New Phytologist, 34/4, pp. 339-344.

López, I. (2009): "Vegetación del manto eólico de Doñana". Memoria de Tesis Doctoral. Universidad de Huelva. 2 tomos. 920 pp.

Mao, K., Hao, G., Liu, J., Adams, R. P. y Milne, R. I. (2010): "Diversification and biogeography of Juniperus (Cupressaceae): variable diversification rates and multiple intercontinental dispersals". New Phytologist, 188: pp. 254-272

Montes, C., Borja, F., Bravo, M. A. y Moreira, J. M. (1998): Reconocimiento biofísico de Espacios Naturales Protegidos. Doñana: una aproximación ecosistémica. Sevilla, C.M.A.

Pérez Latorre, A. V., Galán de la Mera, A. y Carrión, J. (2001): "El papel de las gimnospermas en la vegetación forestal de Andalucía". Revista de Medio Ambiente, 38, Monográfico/13 III Congreso Forestal español. 
Ramírez, L., García-Novo, F., Merino, J. y González-Bernáldez, F. (1977): Sistemas de dunas y arenas estabilizadas de la Reserva Biológica de Doñana. Monografía ICONA 18, Madrid, Publicaciones del Ministerio de Agricultura Pesca y Alimentacion, pp. 159-193.

Rhote, P. L. (1947): "Forêt d'İndochine". Bois et Forêts des tropiques, 1, pp.25-30

Rivas-Martínez, S., Costa, M., Castroviejo, S. y Valdés, B. (1980): "La vegetación de Doñana (Huelva, España)". Lazaroa, 2, pp. 5-190.

Rougerie, G. (1990): Les montagnes dans la biosphère. París, S. Géographie, Armand Colin, 220 pp.

Schnell, R. (1977): Flore et la végétation de l'Afrique tropicale. Tomos 1 y 2. Paris, Gauthier-Villars, 844 pp.

Schnell, R. (1987): La flore et la végétation de l'Amerique tropicale. Tomos 1 y 2. París, Masson. Paris, 928 pp.

Valle, F. (2003): Mapa de Series de Vegetación de Andalucía. Madrid, Edit. Rueda, 131 pp. + Mapa 1:400.000.

Zazo, C., Dabrio, C., Borja, F., Goy, J. L., Lezine, A. M., Lario, J., Polo, M. D. y Hoyos, M. (1999). "Pleistocene and Holocene aeolian facies along the Huelva coast (southern Spain): climatic and neotectonic implications". Geologie in Minjbouw, 77, pp. 209-224.

\section{RESUMEN}

Se estudia el bosque de sabinar o «bosque de naves» de Juniperus phoenicea subsp. turbinata (Guss.) Nyman de la Reserva Biológica de Doñana (RBD), en el entorno de las lagunas de Charco del Toro (CHT) y Navazo del Toro (NVT) utilizando el Método de Inventario de Fanerófitos y Caméfitos (MIFC). Se identifican cinco grupos de sabinares en función de sus elementos constitutivos y la estructura vertical y horizontal (cobertura). Geobotánicamente los sabinares se relacionan con los mantos eólicos litorales de Doñana que tienen una alta conductividad hidráulica y ausencia de una dinámica hídrica subsuperficial. El grupo considerado como formación sabinar-tipo se localiza en el Alto Manto Eólico Seco (AMEs) en el paraje del sabinar de El Ojillo (Grupo 1: NVTv 19).

Palabras Clave: Juniperus phoenicea subsp. turbinata (sabinar) Alto manto eólico seco; transecto lineal; fanerófitos; caméfitos; Doñana.

\section{Abstract}

In this document the savin juniper forest or bosque de naves of Juniperus phoenicea subsp. turbinata (Guss.) Nyman has been characterized in the Doñana Biological Reserve (DRB), by using a plant inventory methodology in linear transects. In this case, the inventory of phanerophytes and chamaephytes (MIFC) in the area surrounding the lagoons of Charco del Toro (CHT) and Navazo del Toro (NVT) ) has been considered. They have identified 5 different groups according to their constituent elements 
and their variables of vertical and horizontal structure (coverage). These are geobotanically related with the aeolian mantle units of Doñana, as well as with the behavior of high transmissivity and hydrodynamic subsurface of the surface formations on which they are distributed. The group considered as savin juniper type formation is located in the Upper Dry Aeolian Mantle (UDAM) where the sabinar de El Ojillo is found (Group 1: NVTV 19).

KeY wORDS: Juniperus phoenicea subsp. turbinata (Guss.) Nyman; Upper Dry Aeolian Mantle (UDAM); linear transect; phanerophytes, chamaephytes; Doñana.

RÉSUMÉ

La forêt de genévriers ou forêt de navires de Juniperus phoenicea subsp. turbinata (Guss.) Nyman a été caractérisée dans la Réserve Biologique de Doñana (RBD) en utilisant une méthodologie d'inventaire végétal en transects linéaires considérant des phanérophytes et des chamaephytes (MIFC) dans le voisinage des lagunes de Charco del Toro (TCS) et Navazo del Toro (NVT). Cinq groupes de genévriers ont été identifiés en fonction de leurs éléments constitutifs et de leurs variables de structure verticale et horizontale (couverture), liés du point de vue géobotanique aux unités de manteaux éoliens de Doñana et au comportement de haute tranmissivité et à la dynamique hydrique du sous-sol des formations superficielles sur lesquelles ils sont distribués. Le groupe considéré comme la formation de genévriers type est situé dans le Haut Manteau Éolien Sec (HMES) dans la contrée du genévrier de El Ojillo (Groupe $1:$ NVTv 19).

Mots Clés: Juniperus phoenicea subsp. turbinata (Guss.) Nyman; Haut Manteau Éolien Sec; transect linéaire; phanérophytes; chamaephytes; Doñana. 